\title{
Biomarkers of fruit and vegetable consumption: findings from the FLAVURS study
}

\author{
D. Alimbetov, Y. N. Jin, T. W. George, M. Chong, J. P. E. Spencer, M. H. Gordon \\ and J. A. Lovegrove \\ Department of Food and Nutritional Science and Institute for Cardiovascular and Metabolic Research (ICMR), \\ University of Reading, Whiteknights, PO Box 226, Reading, Berks RG6 6AP, UK
}

\begin{abstract}
Increased consumption of fruits and vegetables $(\mathrm{F} \& \mathrm{~V})$ has been associated with decreased risk of chronic diseases such as CVD and certain types of cancers ${ }^{(1,2)}$. Daily consumption of five portions $(400 \mathrm{~g})$ or more of $\mathrm{F} \& \mathrm{~V}$ has been recommended by the WHO and the UK's Scientific Advisory Committee of Nutrition (SACN). Yet there is no clear advice on the optimum type or quantity of F\&V for disease prevention. Epidemiological evidence supports the association of high-dietary flavonoid intake and reduced risk of CVD ${ }^{(3)}$. However, dose response, randomised, controlled trials are required to confirm these findings.

FLAVURS (FLAVonoids at University of Reading Study) was a large, parallel, randomised, controlled study with sequential doseresponse increase in F\&V consumption. Subjects were randomised to either a control group (CT) or one of two intervention groups: high flavonoid (HF) or low flavonoid (LF). The groups were balanced for gender, age, BMI, smoking status and consumption of tea and red wine. After a 2-week run-in period, with no dietary changes, participants were randomised to one of the study groups. The CT group continued on their habitual diet, while the HF and LF groups sequentially increased their daily F\&V intake by two portions every 6 weeks to a maximum of six extra portions, with flavonoid-rich or flavonoid-poor $\mathrm{F} \& \mathrm{~V}$, respectively. The primary endpoint measure was vascular function. Fasting blood and $24 \mathrm{~h}$ urine samples were collected at baseline and at weeks 6,12 and 18 for the determination of biomarkers of intake. Eight to twelve randomised $24 \mathrm{~h}$ dietary recalls were analysed using Dietplan6 for the determination of daily nutrient intake.

A total of 154 participants (sixty men, ninety-four women), mean (SEM) age 51 (SE 1) year, and BMI $27.6 \pm 0.4 \mathrm{~kg} / \mathrm{m}^{2} \mathrm{completed} \mathrm{all}$ study visits. Total urinary excretion and dietary flavonoid intake increased in a dose-dependent manner in the HF group $(P=0.0001)$, with values higher than $\mathrm{CT}$ and LF groups $(P>0.0001)$ at 6,12 and 18 weeks. A time-by-treatment interaction for plasma and dietary vitamin $\mathrm{C}$ concentrations were observed $(P=0.0001)$ with the HF and LF groups increasing dose dependently, with levels higher than CT group at all intervention visits. A time-by-treatment interaction was observed for total plasma and dietary carotenoids $(P=0.0001)$ in addition to individual plasma carotenoids: $\alpha$-carotene $(P=0.0001)$; $\beta$-carotene $(P=0.0001) ; \beta$-cryptoxanthin $(P=0.016)$ and lycopene $(P=0.0043)$. There was a significant correlation at all time points between dietary intakes and biomarker concentrations for total flavonoids, which was strongest after two portions for total flavonoids $(r=0.385, P=0.0001)$ and flavonones $(r=0.412, P=0.0001)$ and vitamin $\mathrm{C}$ after two and six portions $(r=0.339, P=0.0001 ; r=0.341, P=0.0001$, respectively). The use of urinary flavonoids, plasma carotenoids and vitamin $\mathrm{C}$ as biomarkers for $\mathrm{F} \& \mathrm{~V}$ intake was supported by data from the FLAVURS study.
\end{abstract}

The project was funded by Food Standard Agency (project no. N02039/F5234012).

1. Kris-Etherton PM, Hecker KD, Bonanome A et al. (2002) Am J Med 113, 71-88.

2. Hodgson JM, Croft KD (2006) J Sci Food Agric 15, 2492-2498.

3. Yeboah J, Crouse JR, Hsu F-C et al. (2007) Circulation 115, 2390-2397. 